\title{
Surprising protective mechanisms against severe forms of COVID-19 infection among Common Variable Immunodeficiency Patients- one center experience.
}

\section{Carina Petricau ( $\nabla$ carina.petricau@yahoo.com )}

Regional Institute of Gastroenterology and Hepatology "Prof Dr O Fodor" Cluj-Napoca, Romania Irena Nedelea

Regional Institute of Gastroenterology and Hepatology "Prof Dr O Fodor" Cluj-Napoca, Romania Diana Deleanu

Regional Institute of Gastroenterology and Hepatology "Prof Dr O Fodor" Cluj-Napoca, Romania

\section{Research Article}

Keywords: common variable immunodeficiency, coronavirus disease 2019 pandemic, cytokine storm, protective mechanisms

Posted Date: August 12th, 2020

DOI: https://doi.org/10.21203/rs.3.rs-57542/v1

License: (c) (1) This work is licensed under a Creative Commons Attribution 4.0 International License.

Read Full License 


\section{Abstract}

In this report we aimed to present the nonthreatening experience of patients diagnosed with Common variable immunodeficiency (CVID) included in the National Rare Disease Program registry and consulted at the Immunology department of the Regional Institute of Gastroenterology and Hepatology "Prof Dr. Octavian Fodor" during the Coronavirus disease 2019 (COVID-19) pandemic as well as to review the current understanding of COVID-19 immunopathology followed by possible protective mechanisms against severe infection in these highly susceptible individuals. We report clinical and laboratory results of patients in a single-center retrospective study after lockdown restrictions were partially lifted (MayJune 2020) and patients were able to come into the hospital for routine check-up and immunoglobulin replacement treatment. Of the 49 patients consulted during this period, we identified only one asymptomatic patient with severe acute respiratory syndrome coronavirus 2 (SARS-CoV-2) infection, supporting recently published data that not all immune compromised patients are at increased risk. According to recent publications the virus induces an inflammatory response leading to a cytokine storm responsible for severe complications. CVID patients seem to be protected from severe forms of this severe virus through reduced viral susceptibility, deficient B lymphocyte response, loss of Interleukin-6 (IL6) receptor and impaired toll-like receptor pathway activation. Despite being at high risk for other infectious disease, in the context of SARS-CoV-2 induced pandemic, CVID patient's lack of immune response is their protection against the dangerous macrophage hyper-activation resulting cytokine storm consequences.

\section{Introduction}

Common variable immunodeficiency (CVID) is a heterogeneous immune disorder characterized by recurrent infections, associated with autoimmune disorders, and increased risk of malignancy; defined by hypogammaglobulinemia involving $\lg G$ and either $\lg A$ and/or IgM deficiency as a result of reduced numbers of isotype-switched memory B cells and consequently absence of plasma cells [1]. Apart from a small subset of identified molecular defects, the main mechanisms accounting for the defective $B$ cell differentiation into plasma cells observed in all cases have remained, for the most part, unknown [2,3]. Besides the lack of immunoglobulin production and its clinical ramifications, failure of $B$ cell development also presents with a few other identified molecular alterations which may in fact prove to be protective in certain viral induced conditions.

Patients with an immune disorder, that can be potentially life-threatening in the face of bacterial pathogens and quality-of-life modifying through progressive chronic disease affecting multiple systems as well as frequently associated with autoimmune and malignant disorders; would qualify as a highly susceptible group of individuals in the current Coronavirus disease 2019 (COVID-19) pandemic. To date however, global surveys designed to collect data on COVID-19 in Primary Immunodeficiency patients do not point to an increased risk of infection, especially not in its severe form. Several cases of SARS-CoV-2 infection have been reported world-wide among those with predominantly humoral primary immunodeficiency, nonethelessremarkable recovery was observed in most cases $[4,5,6]$. 


\section{Methods}

This is a single-center retrospective study of the 49 patients with CVID included in the Romanian National Rare Disease Program registry and consulted at the Immunology and Allergy department of the Regional Institute of Gastroenterology and Hepatology "Prof Dr Octavian Fodor" during the COVID-19 pandemic, from February to June 2020. Collection and analysis of data from clinical, laboratory and diagnostic testing reports was conducted after informed consent was acquired from all participants, followed by literature review of the topic.

\section{Results}

\section{Table 1. Immune status of CVID patients after Covid-19 pandemic peak}

Of the total number of patients (Table 1) consulted during this period, six presented respiratory manifestations (dry/productive cough, sputum production, dyspnea) without positive epidemiological history, thus were not tested; their symptoms being interpreted as manifestations of the chronic lung disorders secondary to CVID. We performed PCR tests for two potentially SARS-CoV-2 infected individuals: patient no. 7 presenting with asthma exacerbation, fever and malaise and patient no. 8 that presented with dyspnea, severe fatigue and myalgia. Both results were negative. One single CVID patient no. 23 from our center was confirmed to have SARS-CoV-2 infection, tested due to direct contact with Covid-19 case, however the patient remained completely asymptomatic, without laboratory tests to suggest a hyper-inflammatory response. Having a state of severe immunodeficiency made it difficult to clear the infection, which lingered for a period of ten weeks, and as expected no specific antibodies could be identified in patient's serum.

Patient immunoglobulin levels (Table1) as expected, were on average, below normal values (exception patients on subcutaneous immunoglobulin replacement therapy that had sufficient supplies during lockdown). IgG (range: $22-1184 \mathrm{mg} / \mathrm{dl}$, median: $524 \mathrm{mg} / \mathrm{dl}$ ), IgA (range: 6-210mg/dl, median:44.6mg/dl), IgM (range: $1-295 \mathrm{mg} / \mathrm{dl}$, median: $40.5 \mathrm{mg} / \mathrm{dl}$ ). Lymphocyte count varied widely (range: $360-82,000 \mathrm{~mm}^{3}$, median: $3,841 / \mathrm{mm}^{3}$ ), with 11 patients presenting lymphopenia.

Noteworthy, among the CVID patients, there is a subgroup that may be at slightly increased risk for COVID-19 infection due to chronic lung disease most commonly expressed in the form of bronchiectasis, as a results of recurrent pulmonary infections and delayed-diagnosis of CVID.

As can be observed in Table 1, sudden lockdown implementationand restricted hospital access left our patients without immunoglobulin replacement therapy for an average of two months resulting in marked hypogammaglobulinemia, putting them at increased risk for various severe infections. Strict social distancing and appropriate hygiene measures were highly recommended to our patients in order to assure their safety until they were able to access immunoglobulin replacement therapy again.

\section{Discussion}


Coronaviruses are a diverse group of single-stranded RNA (ssRNA) viruses which circulate among vertebrates and usually cause mild upper respiratory tract infections in humans [7]. In December 2019, in the city of Wuhan, a novel Betacoronavirus responsible for potentially fatalviral pneumonia was identified, officially named severe acute respiratory syndrome coronavirus 2 (SARS-Co-V-2)[8]. As of July 22,2020 , the virus has caused $15,217,434$ infections world-wide with a case-fatality rate of $4.09 \%$ $(622,454 / 15,217,434)$.

Clinical presentation of SARS-CoV-2 infection is variable, ranging from lack of symptoms to mildmoderate symptoms including fever, dry cough, and fatigue in majority of cases [9], however severe infections can present with acute respiratory distress syndrome and multi-organ failure requiring intensive care unit admission [5]. Less frequent symptoms include headache, sputum production, dyspnea, sore throat, nasal and conjunctiva congestion, myalgia, arthralgia, chills, nausea, vomiting and diarrhea, anosmia and aguesia [10].

Published risk factors for SARS-CoV-2 infections include: advanced age and underlying conditions including Hypertension, Cardiovascular disease, Diabetes, Chronic lung disease, smoking and obesity [11].

The most characteristic laboratory findings among patients infected with the novel virus are: lymphopenia, neutrophilia, high inflammatory markers (C-reactive protein, pro-inflammatory cytokines (IL6), and Ferritin), along withhyper-coagulation state indicated by high D-dimers [11].

\section{Immune mechanisms involved in COVID-19 severity}

The physiological response to viral infections is generally initiated at the cellular level following replication. Transcriptional activation by the intracellular virus leads to the stimulation of two antiviral defense mechanisms. One antiviral response is the induction of type I and type III interferons (IFN) with subsequent upregulation of IFN-stimulated genes and viral control. The second antiviral response iscoordinated by cytokines responsible for the recruitment of specific subsets of white blood cells involved in eradicating the infection [9].

A study that set out to identify the transcriptional signature of SARS-CoV-2, presented data that suggest that the immune response in COVID-19 is imbalanced with regard to controlling viral replicationversus activationof the adaptive immune response [9]. A reduced IFN-I and III response to SARS-CoV-2 was observed, thus viral replication control is weak, while a robust cytokine response (especially IL-6 and IL-1) was noted. The underlying pathophysiology of COVID-19 is still under investigation, but it appears that the virus induces an inflammatory response involving macrophage hyperactivation, leading to a cytokine storm responsible for severe complications [4, 12].

Cytokine release syndrome is a systemic inflammatory response that can be cause by various factors (infections, drugs) characterized by a sharp increase in pro-inflammatory cytokines [8]. Interleukine-6 (IL6) is a multi-faced cytokine with both anti-inflammatory and pro-inflammatory effects that is produced by 
T lymphocytes, endothelial cells, fibroblasts, macrophages and monocytes, activated by Interleukin-1b and tumor necrosis factor-alpha [8]. IL-6 has various biological functions: inducing B-cell proliferation and differentiation to produce antibodies (a process that is absent or defective in agammaglobulinemia and CVID), induces cytotoxic T lymphocyte activity and proliferation, strong inducer of acute-phase reactive proteins (CRP) in the hepatocytes, and is associated with hypergammaglobulinemia [8]. Macrophage activation syndrome-like disease seen in severe COVID-19 cases is also responsible for the commonly observed extensive pulmonary microthrombosis [12]. Blockade of implicated cytokines is responsible for spectacular clinical outcomes withimmunosuppressive targeted monoclonal therapy (anti-IL6 receptor, Tocilizumab) and systemic corticosteroids in moderate to severe cases of COVID-19 [8].

\section{Possible protective mechanisms against severe COVID-19 infection within CVID individuals}

Because immunosuppressive therapies have been shown beneficial in the inflammatory phase of COVID19 , the question that arises is whether certain underlying immune defects identified in CVID can actually be protective?

\section{Limited susceptibility to viral infections}

Patients with predominant antibody immunodeficiency are particularly vulnerable to encapsulated bacterial infections, less prone to viral infections. Patients with agammaglobulinemia are susceptible to a limited number of viral infections (norovirus and enterovirus) while CVID patients are susceptible to rhinoviruses, noroviruses and herpesviruses [5].

\section{Deficient B Lymphocyte responses}

Patients with agammaglobulinemia lack B lymphocytes completely while patients with CVID have variable numbers of dysfunctional $B$ lymphocytes with a marked reduction of mature $C D 27+B$ lymphocytes. Thus we postulate that the lack of appropriate $B$ cell response leads to decreased cytokine release. It has been demonstrated that mature B cells produce IL-6 to drive germinal center formation [5]. COVID-19 treatments might consider the possibility of not only blocking produced cytokines but dampening the inflammatory functions of $\mathrm{B}$ lymphocytes and inhibiting cytokine production by monocytes and dendritic cells [5].

\section{Loss of IL-6 receptor}

Recently, a number of cases with primary immunodeficiency were found to have genetic defects in various components of the IL-6 pathway. Spencer et al. [13] and Nahum et al [14] reported cases with IL-6 receptor loss of function defects. Although these patients lack expression of a function IL-6R chain, it was hypothesized that cells should retain responsiveness to IL-6 through trans-signaling by binding gp130 co-receptor, however this was not the case, resulting in complete loss of IL-6 responsiveness in these patients [14]. Thus, even if IL- 6 is produced by immune cells as a response to infection, a subset of patients with primary immune deficiency demonstrate a complete loss of responsiveness to IL-6. An 
accessible measure to demonstrate lack of physiological IL- 6 activity can be a disproportionate level of low CRP (normally synthesized by hepatocytes response to IL-6) as opposed to high serum IL-6 [14].

\section{Impaired Toll-like Receptor pathway activation}

The Toll-like receptors (TLR) are a family pattern recognition receptors that are essential in the innate immune response, being activated by different types of pathogen ligands in order to clear infections [15]. TRLs which detect viral nucleic acids (TLR3, 7, 8 and 9) are expressed on intracellular endosome membranes. TLR7 responds to single-stranded ribonucleic acid (ssRNA) viruses [15], such as SARS-CoV2. TLR7 is intrinsically expressed on the membranes of endosomes in plasmacytoid dendritic cells and $B$ lymphocytes, which are abundantly present in lung tissue [16]. Signaling in human immune cells by TLR7 has been well-known to trigger production of pro-inflammatory cytokines including TNF-a, IL-6, IL-1b, IL12 and IFT-a [15]. Thus, it is plausible to assume that the well documented cytokine storm partially responsible for severe forms of SARS-CoV-2 infection could also be a result of exaggerated TLR7 activation in lung residing immune cells. It has been demonstrated that certain TLR pathway activation is impaired in CVID, particularly TLR 7 and TLR9 involved in anti-viral innate immune response [2,3].

\section{High dose Immunoglobulin therapy}

Immunoglobulin replacement therapy is the only therapeutic option for CVID and agammaglobulinemia. High does intravenous immunoglobulin (IVIG) was used in all reported cases of COVID-19 in CVID patients with great results. IVIG could also be potentially useful in treating severe COVID-19 patients due to it's immunomodulatory and anti-inflammatory effects but also possibly due to it's content of antibodies to other coronaviruses that cross react with SARS-CoV-2 [4].

\section{Conclusions}

In this article we report the experience of our CVID patients in the COVID-19 pandemic. Our data concludes that individuals with predominant humoral immunodeficiency, particularly CVID, despite being at high risk for other infectious disease, in the context of SARS-CoV-2 induced pandemic, their lack of immune response could potentially be their protection against the dangerous macrophage hyperactivation resulting cytokine storm consequences. Despite the hypothetical paradoxical protection through immune deficiency, continued appropriate hygiene measures such as wearing a facial mask in public places, appropriate hand washing and disinfection as well as sensible social distancing are important precautions for all individuals, especially those with Primary Immunodeficiencies.

\section{Declarations}

\section{Acknowledgements}

None. 


\section{Authorship contributions}

All authors have directly participated in the analysis of current published data, as well as in the planning and writing of the manuscript. All authors have approved the final version submitted.

\section{Ethics approval and consent to participate}

The study was approved by the Ethics Committee of the "Professor Doctor Octavian Fodor" Regional Institute of Gastroenterology and Hepatology, Cluj-Napoca, Romania.

\section{Disclosure of Conflict of interest}

Nothing to disclose.

\section{References}

1. Cunningham -Rundles $\mathrm{C}$, et al. "Clinical manifestations, epidemiology, and diagnosis of common variable immunodeficiency in adults" UpToDate April 2020.

2. Yu JE, Knight AK, Radigan L, Marron TU, Zhang L, et al. "Toll-like receptor 7 and 9 defects in common variable immunodeficiency" J Allergy ClinImmunol. (2009):05.019.

3. Yu JE, Dockray R, Radigan C, Cunningham-Rundles C. "TLR7 Activation is Defective in Common Variable Immunodeficiency” J Allergy ClinImmunol. (2008): vol.121;nr2, p.168-169

4. Aljaberi R, Wishah K "Positive Outcome in a COVID-19 patient with Common Varible Immunodeficiency after IVIG" Annals of Allergy, Asthma and Immunology (2020), doi: https://doi.org/10.1016/j.anai.2020.06.006

5. 5.Quinti I, Lougaris V, Milito C, Cinetto F, Pecoraro A, et al "A possible role for B cells in COVID-19? Lesson from patients with agammaglobulinemia”J Allergy ClinImmunol. (2020)

6. Soresina A, Moratto D, Chiarini M, et al. "Two X-linked agammaglobulinemia patients develop pneumonia as COVID-19 manifestation but recover" Pediatr Allergy Immunol. (2020). https://doi.org/10.1111/pai.13263

7. Weiss RS, Navas-Martin S. "Coronavirus Pathogenesis and the Emerging Pathogen Severe Acute Respiratory Syndrome Coronavirus" MicrobiolMolBiol Rev. (2005): 69(4): 635-664

8. C, Wu Z, LiJ-W, Zhao H, Wang G-Q. “ Cytokine release syndrome in severe COVID-19: interleukin-6 receptor antagonist tocilizumab may be the key to reduce mortality" International Journal of Antimicrobial Agents 55(2020): 105954

9. Blanco-Melo D, Nilsson-Payant BE, Liu W-C, et al. "Imbalance Host Reponse to SARS-CoV-2 drives development of COVID-19" Cell (2020): 181,1036-1045

10. Guan W, Ni Z, Hu Y, Liang C, et al "Clinical Characteristics of Coronavirus Disease 2019 in China" N Engl J Med (2020);382:1708-20 
11. 11.Tu W-J, Cao J, Yu L, Hu X, Liu Q "Clinicolaboratory study of 25 fatal cases of COVID-19 in Wuhan" Intenstive Care Med (2020)46:1117-1120

12. McGonagle D, Sharif $K, O$ 'Regan A, Bridgewood C "The role of cytokine including Interleukin-6 in COVID-19 induced pneumonia and Macrophage activation syndrome-like disease" Autoimunity Reviews 19 (2020): 102537

13. Spencer S, Bal SK, Egner W, Allen HL. "Loss of the interleukin-6 receptor causes immunodeficiency, atopy and abnormal responses" Journal of Experimental Medicine (2019): 216(9):1986-1998

14. Nahum A, Sharfe N, Broides A, Dadi H, et al "Defining the biological responses of IL-6 by the study of a novel IL-6 receptor chain immunodeficiency" J Allergy ClinImmunol (2020);145(3):1011-1014

15. 15.Petes C, Odoardi N, Gee K. " The Toll for Trafficking: Toll-Like Receptor 7 Delivery to the Endosome" Frontiers in Immunology (2017) 8:1075

16. Platinga $\mathrm{M}, \mathrm{Hammad} H$, Lambrecht $B N$ “Origin and functional specializations of $D C$ subsets in the lung." Eur J Immunol (2010);40:2112-2118

\section{Table}

TABLE 1. Immune status of CVID patients consulted after COVID-19 pandemic peak 


\begin{tabular}{|c|c|c|c|c|c|}
\hline $\begin{array}{l}\text { Patient } \\
\text { No }\end{array}$ & $\begin{array}{l}\text { Route } \\
\text { IG }\end{array}$ & $\begin{array}{l}\text { lgG level } \\
(\mathrm{mg} / \mathrm{dl})\end{array}$ & $\begin{array}{l}\text { IgA level } \\
(\mathrm{mg} / \mathrm{ml})\end{array}$ & $\begin{array}{l}\text { lgM level } \\
(\mathrm{mg} / \mathrm{ml})\end{array}$ & $\begin{array}{l}\text { Lymphocyte count } \\
\text { (mm3) }\end{array}$ \\
\hline 1 & SC & 1086 & 6 & 14 & 570 \\
\hline 2 & SC & 745 & 56 & 20 & 1000 \\
\hline 3 & SC & 715 & 18 & 25 & 1980 \\
\hline 4 & IV & 729 & 77 & 17 & 1280 \\
\hline 5 & IV & 658 & 11 & 9 & 1140 \\
\hline 6 & IV & 48 & 7 & 8 & 2500 \\
\hline 7 & IV & 231 & 7 & 8 & 1330 \\
\hline 8 & IV & 714 & 134 & 30 & 2250 \\
\hline 9 & IV & 593 & 94 & 34 & 3400 \\
\hline 10 & IV & 698 & 144 & 205 & 950 \\
\hline 11 & IV & 337 & 100 & 7 & 660 \\
\hline 12 & IV & 445 & 66 & 17 & 1700 \\
\hline 13 & IV & 549 & 19 & 177 & 1620 \\
\hline 14 & SC & 655 & 6 & 4 & 2060 \\
\hline 15 & IV & 614 & 115 & 116 & 1700 \\
\hline 16 & IV & 466 & 36 & 7 & 82000 \\
\hline 17 & SC & 579 & 8 & 6 & 14250 \\
\hline 18 & IV & 285 & 7 & 36 & 940 \\
\hline 19 & IV & 684 & 13 & 23 & 2510 \\
\hline 20 & SC & 823 & 7 & 3 & 1830 \\
\hline 21 & SC & 834 & 83 & 126 & 1450 \\
\hline 22 & IV & 362 & 15 & 9 & 6230 \\
\hline 23 & IV & 55 & 8 & 4 & 1110 \\
\hline 24 & IV & 498 & 71 & 98 & 2270 \\
\hline 25 & IV & 88 & 6 & 3 & 1510 \\
\hline 26 & IV & 22 & 7 & 5 & 1600 \\
\hline 27 & IV & 394 & 65 & 64 & 1000 \\
\hline 28 & SC & 680 & 6 & 1 & 740 \\
\hline
\end{tabular}




\begin{tabular}{|c|c|c|c|c|c|}
\hline 29 & IV & 566 & 75 & 9 & 1850 \\
\hline 30 & IV & 1184 & 210 & 295 & 1530 \\
\hline 31 & SC & 550 & 7 & 3 & 950 \\
\hline 32 & SC & 605 & 19 & 23 & 1500 \\
\hline 33 & IV & 602 & 158 & 37 & 1400 \\
\hline 34 & IV & 431 & 42 & 7 & 1070 \\
\hline 35 & IV & 766 & 92 & 90 & 1200 \\
\hline 36 & IV & 355 & 7 & 77 & 1320 \\
\hline 37 & IV & 120 & 8 & 5 & 2550 \\
\hline 38 & IV & 164 & 8 & 5 & 1550 \\
\hline 39 & SC & 699 & 6 & 5 & 1930 \\
\hline 40 & IV & 757 & 58 & 217 & 360 \\
\hline 41 & IV & 451 & 12 & 10 & 1880 \\
\hline 42 & SC & 747 & 51 & 23 & 13670 \\
\hline 43 & IV & 605 & 175 & 61 & 1840 \\
\hline 44 & SC & 885 & 7 & 4 & 1250 \\
\hline 45 & IV & 63 & 7 & 6 & 1670 \\
\hline 46 & IV & 335 & 8 & 1 & 2680 \\
\hline 47 & SC & 380 & 12 & 6 & 2200 \\
\hline 48 & IV & 588 & 25 & 22 & 1700 \\
\hline 49 & IV & 284 & 8 & 2 & 2530 \\
\hline
\end{tabular}

